\title{
CTLA- 4 correlates with immune and clinical characteristics of glioma
}

\author{
Fangkun Liu', Jing Huang ${ }^{2,3}$, Xuming Liư ${ }^{4}$, Quan Cheng ${ }^{1}$, Chengke Luo ${ }^{1}$ and Zhixiong Liu ${ }^{1 *}$ (D)
}

\begin{abstract}
Background: CTLA-4 is a well-studied immune checkpoint protein that negatively regulates $T$ cell-mediated immune responses. However, the expression of CTLA-4 in glioma and the effects of CTLA-4 on prognosis in patients with glioma have not yet been examined.

Methods: We investigated the protein level of CTLA-4 in human glioma samples, extracted genetic and clinical data from 1024 glioma patients to characterize CTLA-4 expression and its relationship with immune functions in gliomas. $R$ language was used for statistical analysis.

Results: Higher CTLA-4 expression was found in patients with higher grade, isocitrate dehydrogenase (IDH)-wildtype, and mesenchymal-molecular subtype gliomas than in patients with lower grade, IDH-mutant, and other molecular subtype gliomas. Further analysis showed that there was a strong positive correlation between CTLA-4 and the specific marker gene expression of immune cells, including $C D 8^{+} T$ cells, regulatory $T$ cells, and macrophages in both databases, suggesting that higher CTLA-4 expression in the glioma microenvironment induced greater immune cell infiltration compared with that in gliomas with lower CTLA-4 expression. We further explored the associations between CTLA-4 and other immune-related molecules. Pearson correlation analysis showed that CTLA-4 was associated with PD-1, CD40, ICOS, CXCR3, CXCR6, CXCL12 and TIGIT. Patients with glioma with lower CTLA-4 expression exhibited significantly longer overall survival. Thus, these findings suggested that increased CTLA-4 expression conferred a worse outcome in glioma.
\end{abstract}

Conclusions: In summary, our findings revealed the expression patterns and clinical characteristics of CTLA-4 in glioma and may be helpful for expanding our understanding of antitumor immunotherapy in gliomas.

Keywords: Immune checkpoint, CTLA-4, Immune response, Glioma, Prognosis

\section{Background}

Glioma is the most common primary malignant brain tumor in adults and is associated with a poor clinical course and prognosis. Despite improvements in surgical resection techniques, chemotherapy, and radiotherapy, the median overall survival (OS) of patients with glioma remains dismal. The discovery of the intracranial lymphatic system has challenged the classical dogma that

*Correspondence: zhixiongliu@csu.edu.cn

1 Department of Neurosurgery, Xiangya Hospital, Central South University (CSU), 87 Xiangya Rd, Changsha 410008, Hunan, China

Full list of author information is available at the end of the article the central nervous system (CNS) is immune-privileged and lacks immunosurveillance, which also supports the potential efficacy of immunotherapy in the treatment of glioma [1-4]. The recent striking clinical success of immune checkpoint inhibitors in other advanced cancers has encouraged the exploration of immune checkpoint blockade therapy in patients with glioma $[5,6]$. A variety of clinical trials targeting programmed death (PD)-1/PDligand 1 (PD-L1), and cytotoxic T-lymphocyte antigen (CTLA)-4 have been investigated in patients with glioma to promote the robust antitumor $\mathrm{T}$ cell response [7].

As a well-studied immune checkpoint protein, CTLA-4 plays a crucial role in the tumor immunoreaction process. 
CTLA-4 is a major negative regulator of T-cell activation that interrupts costimulatory signaling and functions provided by cluster of differentiation (CD) 28:B7 binding [7]. Compared with the costimulatory molecule CD28, CTLA-4 has a 10- to 20-fold higher affinity to its ligands B7-1 (CD80) and B7-2 (CD86) [8]. By blocking the interaction between CTLA-4 and its ligands expressed by antigen presenting cells, inhibitors of CTLA-4 can block the inhibitory immune signal and restore anticancer immune responses. Monoclonal antibodies as CTLA-4 inhibitors were first reported in 1996 and have led to suppression of murine allergic contact dermatitis [9]. Inhibitors of CTLA- 4 have also been shown to have remarkable success in clinical cancer immunotherapy in recent years. Ipilimumab is a fully humanized IgG1 subclass monoclonal antibody against CTLA-4 and was approved for melanoma therapy by the US Food and Drug Administration in 2011. Current trials are evaluating the use of this antibody for additional oncological indications. Another humanized anti-CTLA-4 antibody, tremelimumab, has been shown to elicit favorable responses in clinical trials against different tumor types. Combined with immune checkpoint inhibitors targeting PD-1/PD-L1, antibodies that block CTLA-4 may have applications as immunotherapies for the treatment of various malignancies, including glioma.

Several studies of immune checkpoint inhibitors targeting CTLA-4 have demonstrated promising benefits in patients with glioma [7]. The first large phase III trial of ipilimumab (a CTLA-4 inhibitor) plus nivolumab (a PD-1 inhibitor) in recurrent World Health Organization (WHO) grade IV glioma (glioblastoma) (NCT02017717) was initiated in 2014. With so many clinical trials, a comprehensive analysis of CTLA-4 expression will be required to identify the enrichment criteria of CTLA-4 in glioma clinical trials [10]. CTLA-4 was reported to highly expressed in high grade gliomas [11]. Doucette et al. [12] have analyzed the expression patterns of distinct glioma antigens and immune genes including CTLA-4 in different GBM subtypes using 544 samples from TCGA dataset. The mRNA level of CTLA-4 was higher in mesenchymal subtype compared with other GBM subtypes. However, more detailed and comprehensive reports of CTLA-4 expression in glioma is lacking.

Accordingly, in this study, we examined CTLA-4 expression in glioma specimens (WHO grade II-IV), explored the molecular and clinical characteristics of CTLA-4 in glioma by analyzing RNA-seq data from two databases (The Cancer Genome Atlas [TCGA] database and the Chinese Glioma Genome Atlas [CGGA] database). We expect that our findings will help facilitate the development of potential anti-CTLA-4 treatments in glioma.

\section{Methods}

\section{Patients and samples}

We used formalin-fixed and paraffin-embedded (FFPE) glioma specimens to detect the expression of CTLA-4. 58 glioma specimens from adult patients who underwent neurosurgical resection of gliomas at the Department of Neurosurgery, Xiangya Hospital were included in the analysis. We also included three additional normal brain tissues. Historical diagnosis of glioma was performed according to WHO classification. In total, 61 specimens were analyzed, including three normal brain tissues, 20 tumor specimens of glioma grade I-II, 12 tumor specimens of glioma grade III, and 26 tumor specimens of glioma grade IV. The study was approved by the ethics committee of the Xiangya Hospital, Central South University.

The RNA sequencing data and related clinical information were obtained from TCGA (http://cancergeno me.nih.gov/) databases and CGGA (http://www.cgga.org. $\mathrm{cn} /$ ) databases. 699 glioma samples (WHO grade II-IV) from TCGA database and 325 glioma samples (WHO grade II-IV) from CGGA database were included in our analysis. The IDH mutation data from TCGA were generated using whole exon sequencing or pyrosequencing. The IDH mutation data from CGGA were examined by pyrosequencing. Survival analysis in both databases were calculated from either the date of diagnosis or the start of treatment for a disease until death or last follow-up examination.

\section{Statistical analysis}

Statistical analysis was mainly performed using $\mathrm{R}$ language (version 3.5.1; http://www.R-project.org) [13]. Several publicly available packages have been employed for figure generation. The heatmap was generated using the "pheatmap" package [14], Correlation between CTLA-4 and other checkpoint members were analyzed by Spearman correlation using the "circlize" package [15]. ROC curves were derived using the "pROC" package [16]. The Cox regression analysis was performed using the "survival" package [17], survival differences were compared by Kaplan-Meier method. All statistical tests were twotailed and a $\mathrm{p}<0.05$ was considered to be significant in our study.

\section{Results}

\section{CTLA-4 expression status in glioma}

We analyzed the expression of CTLA-4 in glioma according to WHO grade, IDH mutation status, and different subtypes in TCGA and CGGA databases. Gene expression profiling data in both databases were log-transformed for further analysis. In TCGA database, higher expression of CTLA-4 was detected in higher grade 
glioma samples. The highest CTLA-4 expression was found in WHO grade IV glioma (glioblastoma; Fig. 1a). We also validated our findings in CGGA database; similar to the results from TCGA database, higher expression of CTLA-4 was observed in grade IV glioma compared with that in grade II and grade III glioma $(p<0.01)$. The difference in CTLA-4 expression between grade II and grade III gliomas was not significant (Fig. 1b). These results indicated that CTLA-4 expression was positively correlated with pathogenic condition and malignancy in glioma. Further protein expression pattern of CTLA-4 in glioma also suggested that CTLA-4 expression was detected in high-grade glioma samples (Fig. 1c) (Additional file 1: Table S1).

Mutations in IDH are well-known molecular markers for the classification of different subtypes of gliomas [18]. Therefore, we examined CTLA-4 expression according to IDH mutation status in TCGA and CGGA databases. Gliomas possessing IDH mutations expressed lower CTLA-4 in TCGA database (Fig. 1d). Consistent with these findings, in CGGA database, CTLA-4 expression was significantly higher in IDH-wild-type gliomas than IDH-mutated gliomas (Fig. 1e).

Different molecular subtypes of glioma are associated with different responses to treatment and prognoses
[19]. To further characterize the relationship between CTLA-4 and TCGA-defined molecular subtypes, we measured CTLA-4 levels in different glioma subtypes. CTLA-4 expression in mesenchymal-molecular subtype glioma was significantly higher than in the other three subtypes in both databases (Fig. 2a, b). Receiver operating characteristic (ROC) curves were then generated using CTLA-4 expression and mesenchymal-molecular subtype to examine the predictive value in the databases we used. The areas under the curve were $80.8 \%$ and $78.2 \%$ for TCGA and CGGA databases, respectively (Fig. 2c, d). Our findings showed that CTLA-4 may serve as a good predictor for mesenchymal subtype glioma.

\section{Association between CTLA-4 expression and immune cell populations}

Infiltration of immune cells plays a crucial role in immune and inflammatory responses against tumor cells [20]. As described by Etienne Becht et al. [21], we used the microenvironment cell populations-counter method to evaluated the association between CTLA-4 and immune cell populations in TCGA and CGGA databases. A detailed list of each immune cell type and related specific biomarkers is given in Additional file 2: Table S2. Six immune cells were analyzed, including
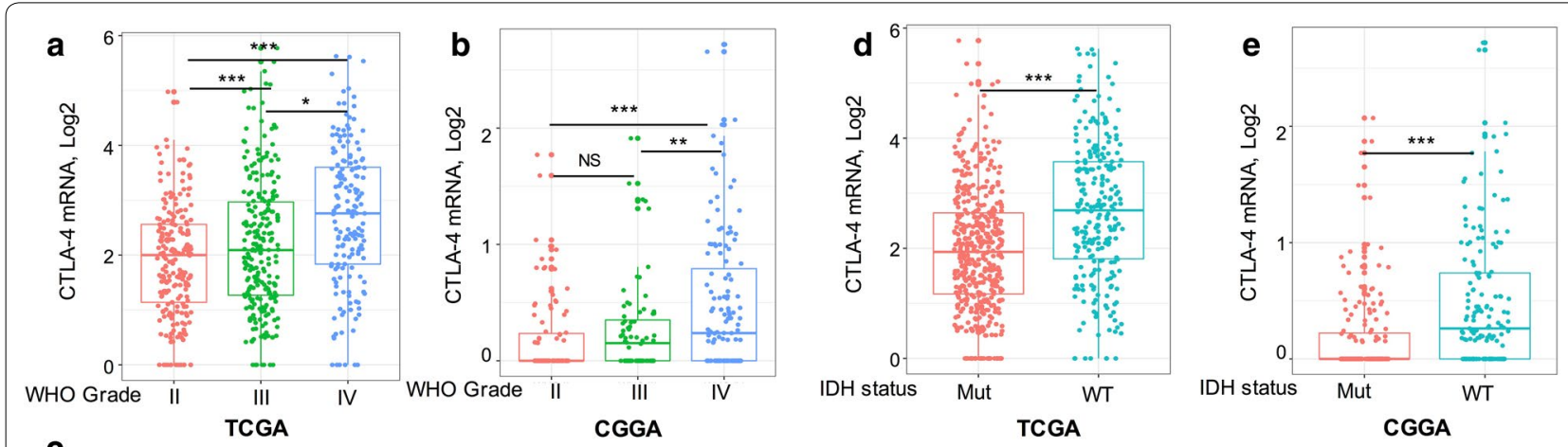

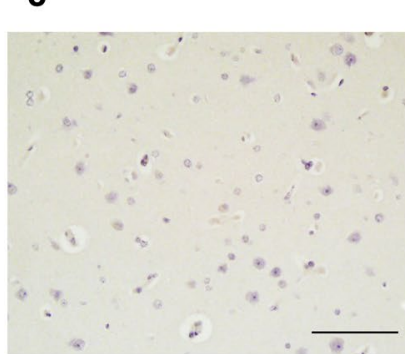

Normal (0/3, 0\%)

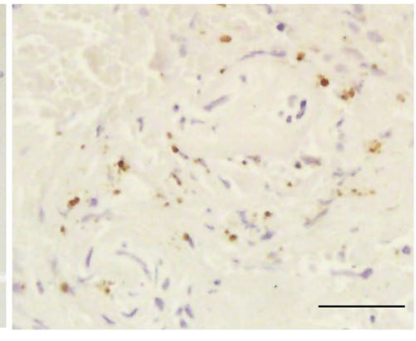

Glioma grade I-II $(3 / 20,15.0 \%)$

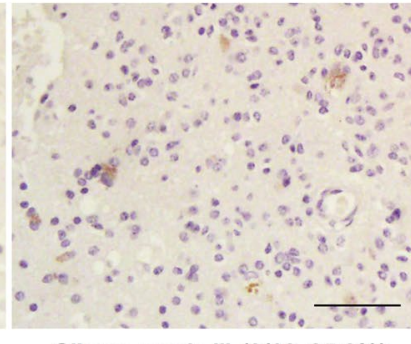

Glioma grade III $(3 / 12,25.0 \%)$

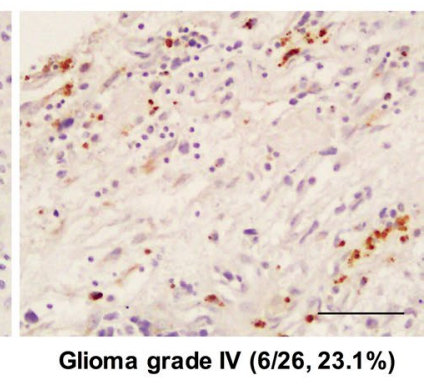

Fig. 1 Relationship between CTLA-4 expression and clinical parameters of glioma. CTLA-4 expression in gliomas according to WHO grade status in the TCGA (a) and CGGA (b) databases. c Glioma tissues of different grades were immunostained for CTLA-4 using anti- CTLA-4 antibody and 3,30-diaminobenzidine (DAB; brown). Haematoxylin was used for nuclear counterstaining (blue). Numbers in parentheses indicate number and percentage of CTLA-4-positive samples out of number of total samples. Scale bars, $100 \mathrm{~mm}$. CTLA-4 expression in gliomas according to IDH status in TCGA (d) and CGGA databases (e). * means $p$ value $<0.05$, ** means $p$ value $<0.01$, *** means $p$ value $<0.001$ 

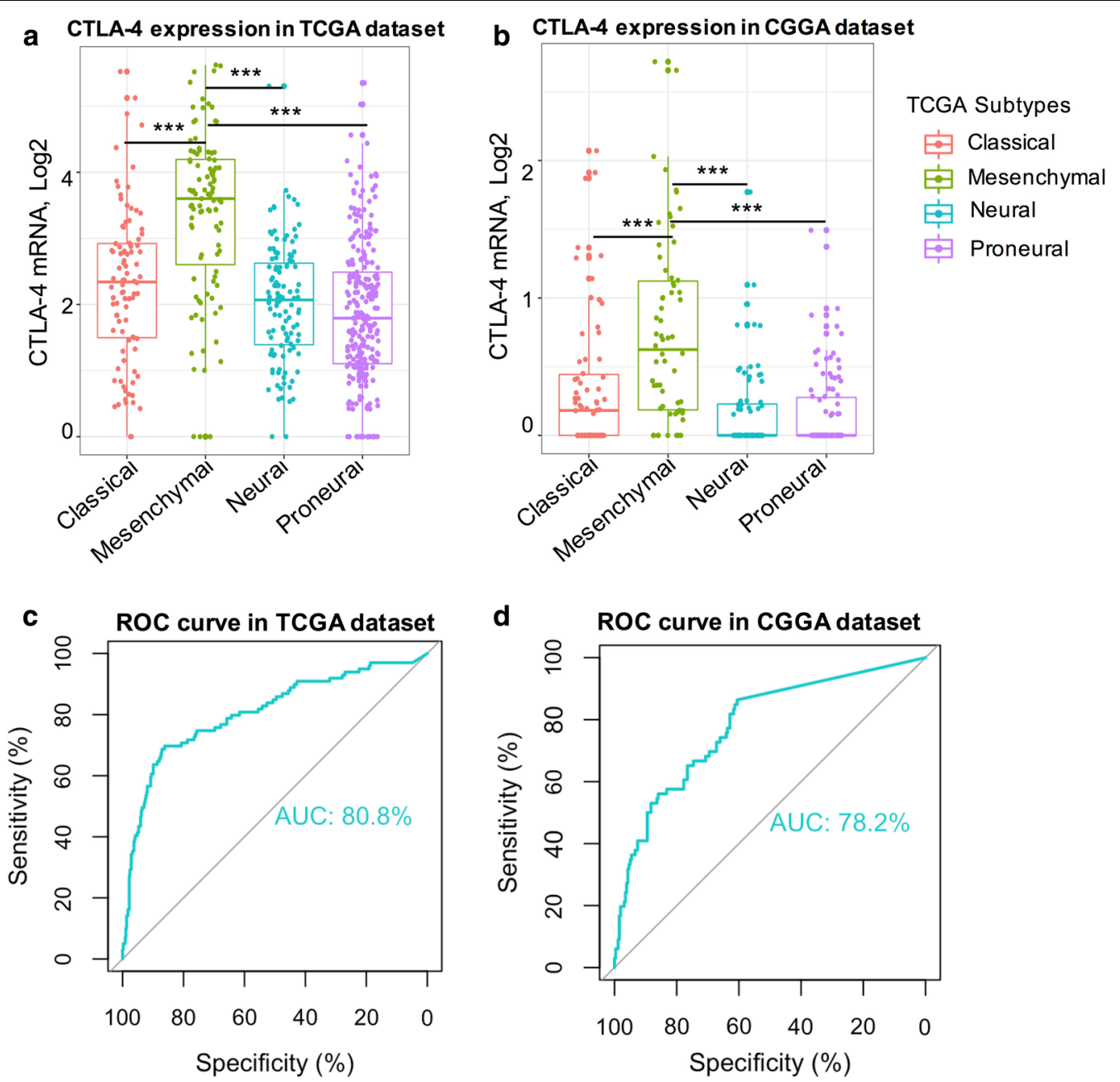

Fig. 2 Relationship between CTLA-4 expression and glioma molecular subtypes. CTLA-4 expression pattern in different molecular subtypes of gliomas in TCGA database (a) and the predictive value analyzed by ROC curve analysis (c); CTLA-4 expression pattern in different molecular subtypes of gliomas in CGGA databases (b) and the predictive value analyzed by ROC curve analysis (d)

$\mathrm{CD}^{+} \mathrm{T}$ cells, regulatory $\mathrm{T}$ cells (Tregs), natural killer cells, tumor-associated macrophages, myeloid-derived suppressor cells, and neutrophils. As shown in the generated heatmap, CTLA-4 was positively correlated with the specific marker gene expression of all six immune cell types examined, particularly in TCGA database (Fig. 3a, b). Detailed information regarding the $\mathrm{r}$ and $p$ values between CTLA-4 and each immune cell type is given in Additional file 3: Table S3. The specific marker gene expression of all six immune cell types was significantly positively correlated with CTLA-4 expression in TCGA and CGGA databases. Moreover, there was a strong positive correlation between CTLA- 4 and the following immune cells in both databases: $\mathrm{CD}^{+} \mathrm{T}$ cells $(\mathrm{r}=0.65$ in TCGA database, $r=0.62$ in CGGA database), Tregs $(r=0.70$ in TCGA database, $r=0.66$ in CGGA database), and macrophages $(r=0.60$ in TCGA database, $r=0.63$ in CGGA database) (Additional file 4: Figure S1). These results indicated that higher CTLA-4 expression in the glioma microenvironment resulted in greater immune cell infiltration compared with glioma with lower CTLA-4 expression.

\section{Correlation between CTLA-4 and immune-related molecules}

Combination therapies of different immune checkpoints inhibitors have shown great benefits compared with mono checkpoint therapy; indeed, combination therapies may yield higher anticancer immune responses and reduced immune-related adverse events [7, 22]. Researchers have shown that combination therapy with the CTLA-4 inhibitor 


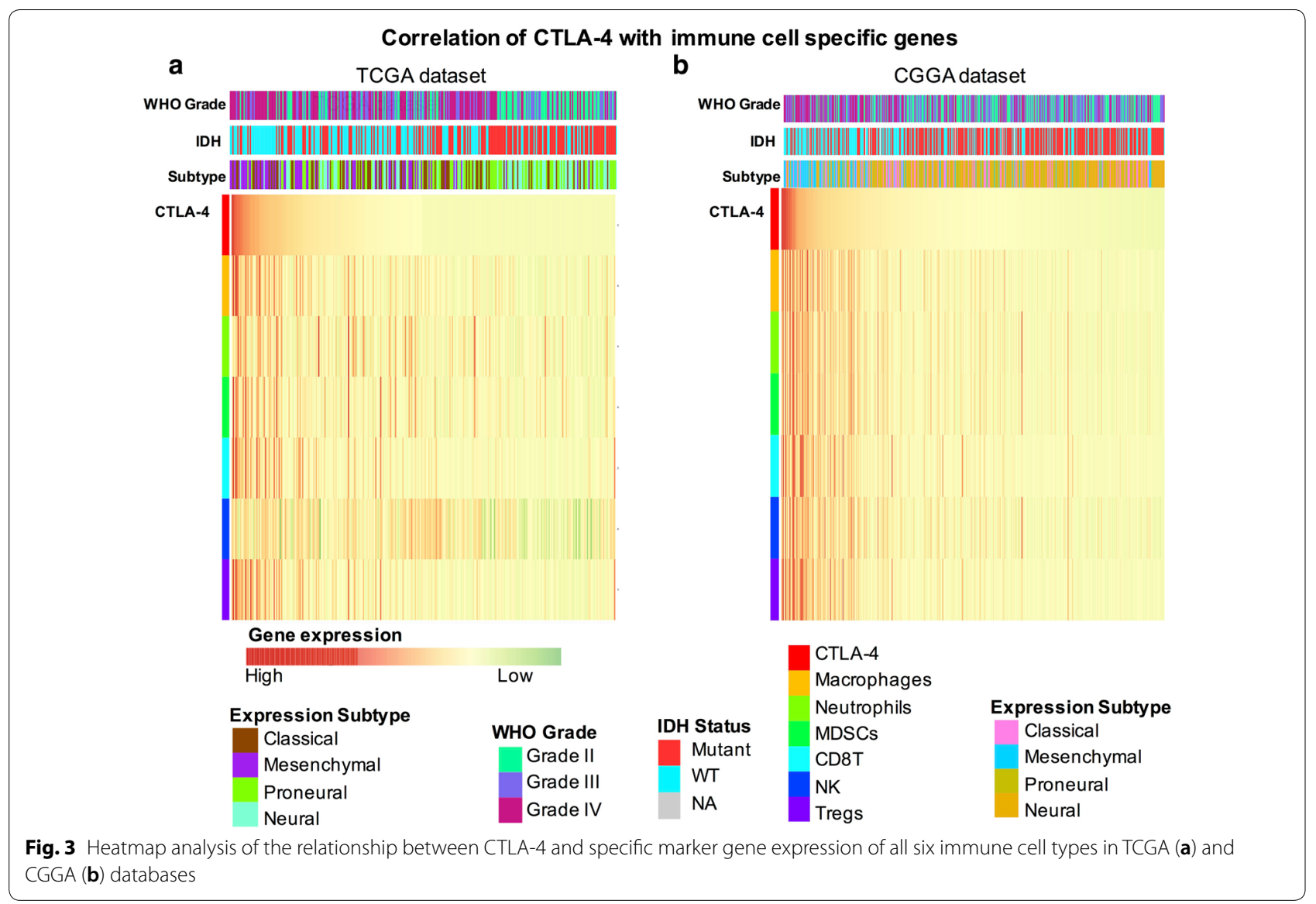

nivolumab and the PD-1 inhibitor ipilimumab was more effective and resulted in significantly longer progression-free survival compared with monotherapy [23]. Therefore, we explored the correlation between CTLA-4 and other immune-related molecules. First, we examined the relationships between CTLA-4 and PD-1 [24], CD40 [25], indoleamine 2,3-dioxygenase 1 (IDO1) [26], and inducible T-cell costimulator (ICOS) [27], which have been reported in preclinical or clinical studies to be combined with CTLA-4 to enhance immunotherapy efficiency. Through Pearson correlation analysis, CTLA-4 was found to be significantly correlated with PD-1, CD40, and ICOS in TCGA and CGGA databases (Fig. 4a, b). CTLA-4 showed stronger associations with PD-1, CD40, and ICOS in patients with glioblastoma in both databases (Fig. 4c, d). Moreover, we analyzed the relationships between CTLA-4 and other immune-related molecules and showed that CTLA-4 was tightly associated with C-X-C motif chemokine receptor (CXCR) 3, CXCR6, and C-X-C motif chemokine ligand (CXCL) 12 in both TCGA and CGGA databases (Fig. 5a, b). Subsequent analysis showed that CTLA-4 was tightly associated with
CXCR3, CXCR6, CXCL12, and T cell immunoreceptor with Ig and ITIM domains (TIGIT) in patients with glioblastoma (Fig. 5c, d).

\section{Prognostic value of CTLA-4 in glioma}

Based on our findings in TCGA and CGGA databases, higher expression of CTLA-4 was observed in higher grades of glioma, highlighting the possible relationship of CTLA-4 expression with poorer prognosis. Therefore, we divided patients with glioma into low- and high-expression groups to evaluate the prognostic value of CTLA-4. As shown in Fig. 6, patients with glioma with lower CTLA-4 expression exhibited significantly longer OS compared with patients with glioma with higher CTLA-4 expression in both TCGA and CGGA databases (Fig. 6a, b). Consequently, we also explored the prognostic value of CTLA-4 expression in patients with low-grade gliomas (LGGs). Similar Kaplan-Meier curves were observed in patients with LGGs (Fig. 6c, d). These findings indicated that increased CTLA-4 expression conferred worse outcomes in patients with glioma. 


\section{Correlation of CTLA-4 with other immune related molecules in glioma}

a

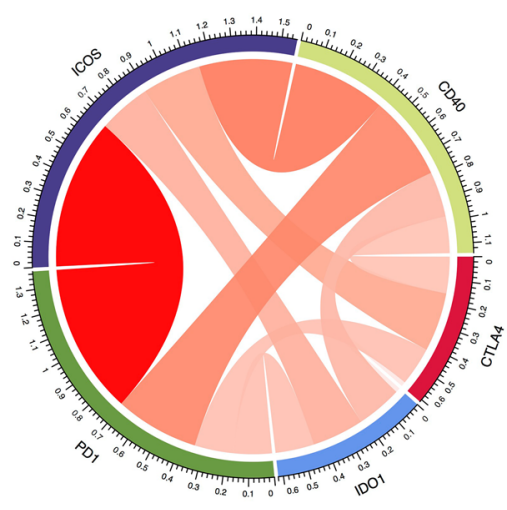

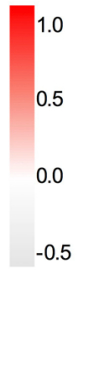

b CGGAdataset

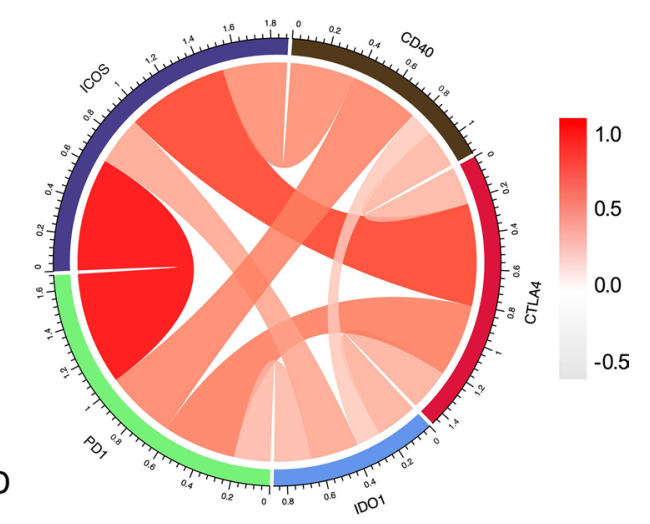

\section{Correlation of CTLA-4 with other immune related molecules in GBM}

C

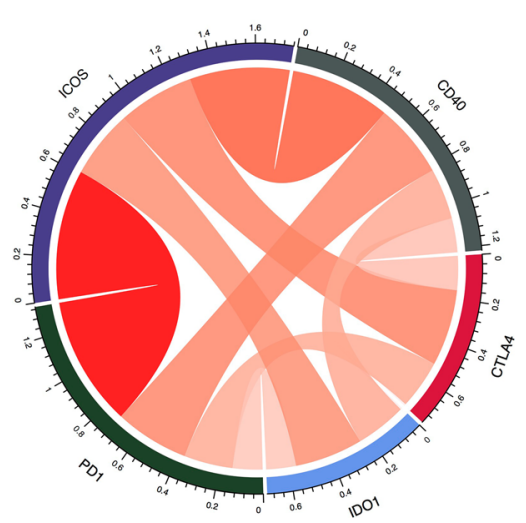

d

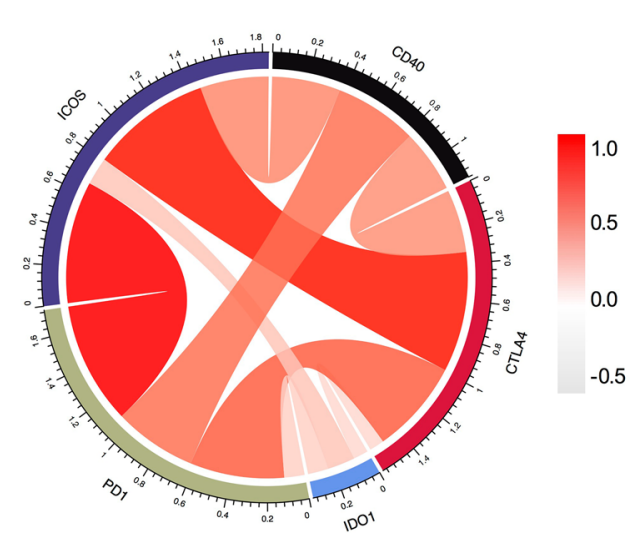

Fig. 4 Association between CTLA-4 and immune related molecules in glioma. The correlations between CTLA-4 and PD-1, CD40, ICOS, IDO1 in glioma (a) and GBM (c) were analyzed based on TCGA database. The correlations between CTLA-4 and PD-1, CD40, ICOS, IDO1 in glioma (b) and GBM (d) were analyzed based on CGGA database

\section{Discussion}

Immune checkpoint therapies targeting PD-1/PD-L1 and CTLA-4 have shown striking success against a variety of solid tumors. The genetic and clinical characteristics of PD-1/PD-L1 in gliomas have been explored, and the PD-1/PD-L1 axis has been shown to have malignant biological functions in glioma [28, 29]. Similar expression patterns for CTLA-4 were observed in our study. We found that CTLA-4 tended to be expressed at higher level in higher grade gliomas, IDHwild-type gliomas, and mesenchymal-subtype gliomas. Similar expression patterns were also reported for other immune checkpoints such as PD-L1 [29], B7-H3 [26], TIM-3 [27], CD155 [30], and PTPN2 [28] in glioma. Besides, Kaffes et al. found highest immune cells presence in mesenchymal GBM compared with other subtypes [31]. These results suggested mesenchymalsubtype gliomas may be more amenable to immunotherapy. The expression pattern of CTLA-4 in our study can be a supplement to the existing findings.

Subsequent analyses of the relationships between CTLA-4 expression and immune cells showed that the specific marker gene expression of all six immune cell types was significantly positively correlated with CTLA-4 expression. Moreover, higher CTLA-4 expression was associated with worse prognosis in patients with gliomas.

CTLA-4 is expressed on activated conventional $\mathrm{T}$ cells and $\mathrm{CD} 4^{+} \mathrm{Foxp}^{+}$Tregs and suppresses antigenspecific T-cell activation [7]. Previous studies have 


\section{Correlation of CTLA-4 with other immune related molecules in glioma}

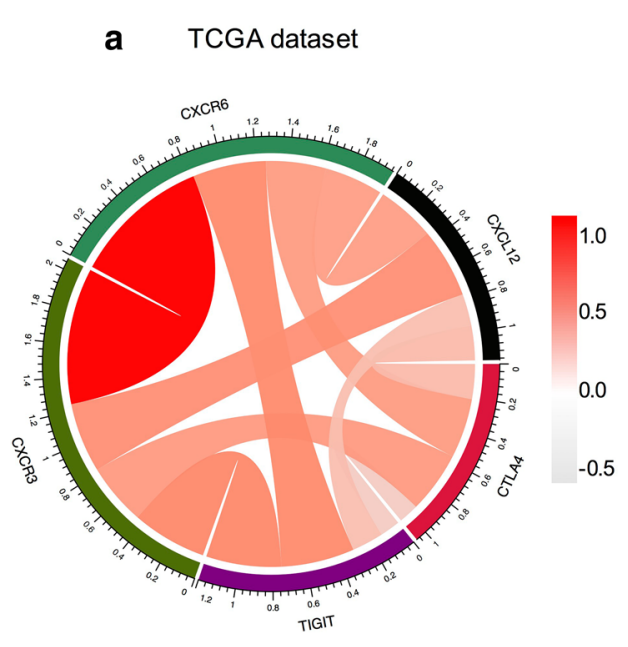

b CGGAdataset

\section{Correlation of CTLA-4 with other immune related molecules in GBM}
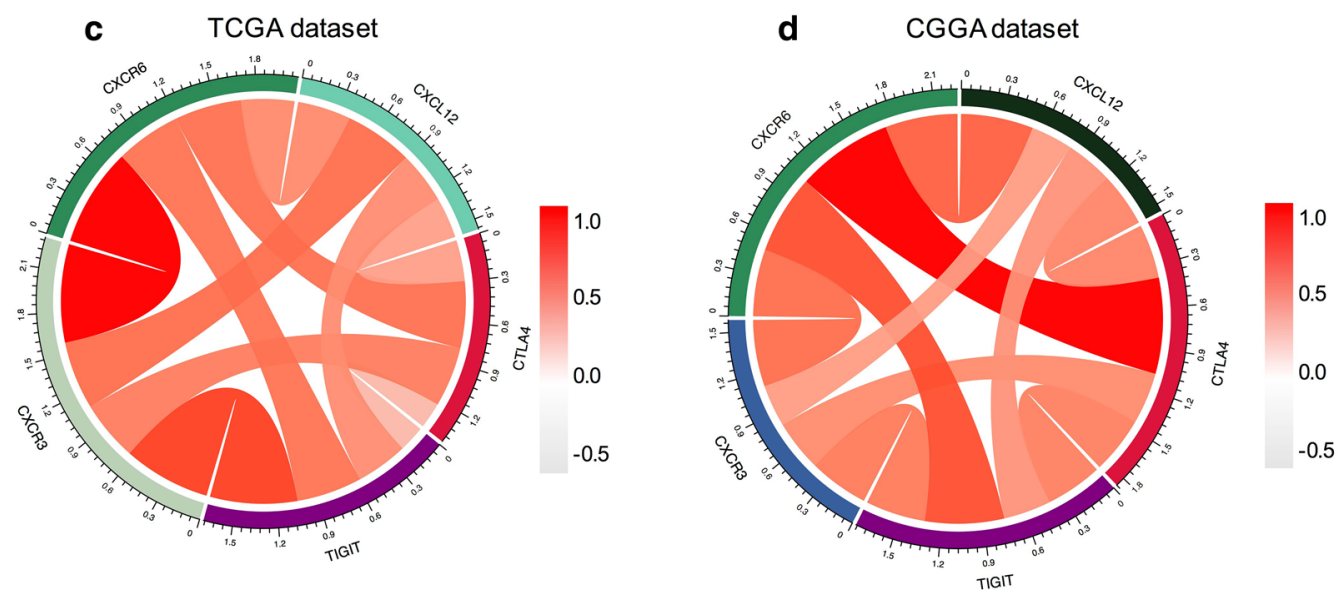

Fig. 5 Association between CTLA-4 and immune related molecules in glioma. The correlations between CTLA-4 and CXCR3, CXCR6, CXCL12, TIGIT in glioma (a) and GBM (c) were analyzed based on TCGA database. The correlations between CTLA-4 and CXCR3, CXCR6, CXCL12, TIGIT in glioma (b) and GBM (d) were analyzed based on CGGA database

investigated CTLA-4 expression in patients with radically resected stage I-III non-small cell lung cancer (NSCLC) using tissue microarray immunohistochemistry. Higher CTLA-4 expression was found in nonsquamous NSCLC than in squamous NSCLC and in low Ki-67-expressing tumors [32]. CTLA-4 expression was also evaluated in $\mathrm{CD}_{3}{ }^{+}$cells from patients with myelodysplastic syndrome, chronic myelomonocytic leukemia, and acute myeloid leukemia. Aberrant upregulation was observed in $8 \%$ of patients [33]. Another study showed that all melanoma cell lines analyzed expressed CTLA-4, and approximately 67\% of melanoma specimens expressed CTLA-4 at different levels of intensity [34]. Other studies have examined the expression of CTLA-4 in nonlymphoid cells, suggesting that this molecule may be involved in more functions besides $\mathrm{T}$ cell response inactivation [35]. Fong et al. [36] analyzed the expression of CTLA-4 on peripheral blood lymphocyte subsets in GBM patients treated with DC vaccination and found CTLA-4 can predict survival in GBM patients. Higher expression of CTLA-4 was correlated with shorter survival after treatment. However, researchers also found blockade of immune-checkpoint inhibitors failed to recapitulate corresponding biomarkers-based clinical predictions using an orthotopic GL261-glioma mice model, which need further research to assess the applicability for clinical use [37].

Preclinical and clinical studies have demonstrated that combination of CTLA-4 and some immune-related 

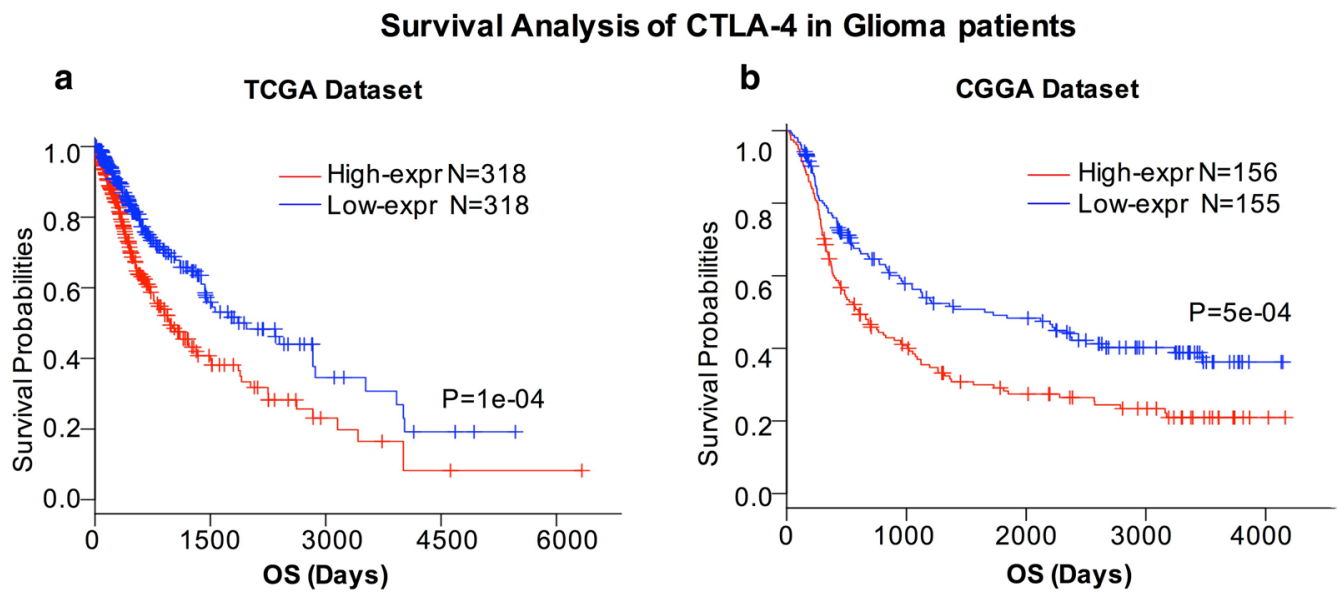

Survival Analysis of CTLA-4 in LGG patients
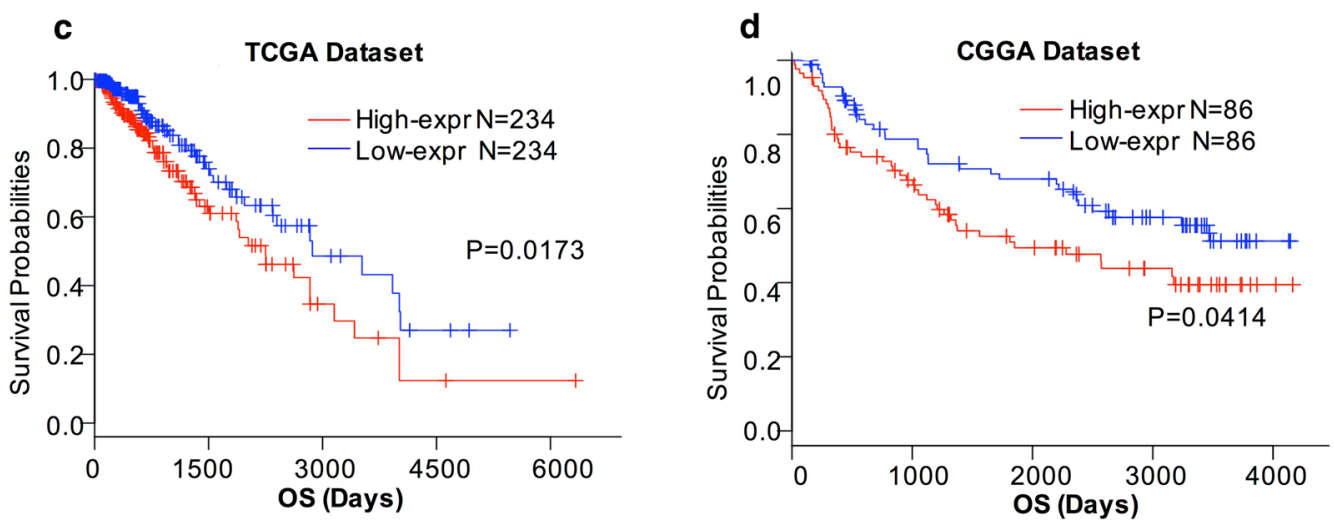

Fig. 6 Survival analysis of glioma based on CTLA-4 expression. Higher CTLA-4 expression is associated with worse overall survival (OS) in patients with glioma and LGG based on TCGA database $(\mathbf{a}, \mathbf{c})$. Higher CTLA-4 expression is associated with worse overall survival (OS) in patients with glioma and LGG (b, d) in CGGA database

molecules may enhance antitumor immunity. Saha et al. [38] showed that the triple combination of OHSV G47A expressing murine IL-12 with anti-CTLA-4 and antiPD-1 antibodies extended survival with no pathological symptoms up to 9 months in a mouse glioma model. Field et al. [39] found blocking CTLA-4 priming with a whole cell vaccine eradicated tumor and prolonged survival in an orthotopic glioma model. Therefore, the relationships of CTLA-4 with PD-1, IDO1, CD40, and ICOS were evaluated, which have been reported to enhance immunotherapy efficiency in combination with CTLA-4. Blockade Inhibition of both PD-1 and CTLA-4 resulted in effectively restoration of T-cell proliferation and secretion of effector cytokines in tumors [40]. Additionally, clinical trials of anti-CTLA-4 (ipilimumab) and antiPD-1 (nivolumab) have also been performed in patients with glioma [7]. The combination of anti-CTLA-4 monoclonal antibodies, anti-PD-L1 monoclonal antibodies, and the IDO inhibitor INCB23843 in the murine B16.SIY melanoma model showed markedly improved tumor control over single-drug treatment [26]. Moreover, a booster vaccination plus combinational treatment with CD40 stimulation and CTLA- 4 inhibition resulted in complete tumor regression in a murine melanoma model [25]. ICOS belongs to the CD28/CTLA-4/B7 immunoglobulin superfamily and has been shown to play diverse roles in optimal antitumor responses mediated by anti-CTLA-4 therapy $[27,41]$. In our study, we found that CTLA-4 was significantly correlated with PD-1, CD40, and ICOS in patients with glioma and glioblastoma. Recent studies have demonstrated that targeting the CXCR4/CXCL12 axis can restore sensitivity to CTLA4 and PD-1 checkpoints inhibitors [42]. Therefore, in this study, we analyzed the associations of CTLA-4 with CXCL12, CXCR3, CXCR6, and TIGIT, a new promising immune checkpoint-related protein [43]. We found that all of these molecules were tightly associated with CTLA-4 in patients with glioma. These results indicated the combination 
with these molecules can potentially enhance the efficacy of CTLA-4 blockade in cancer immunotherapy. Further researches can be performed to study the synergistic effect of these molecules.

\section{Conclusion}

In summary, we investigated the associations of CTLA-4 expression with clinicopathological findings and IDH mutation status in gliomas. Moreover, we found that CTLA-4 was positively correlated with other immunerelated proteins in glioma. Additional studies are needed to further explore the molecular mechanisms mediating CTLA-4 expression in gliomas and responses to antiCTLA-4 therapy.

\section{Supplementary information}

Supplementary information accompanies this paper at https://doi. org/10.1186/s12935-019-1085-6.

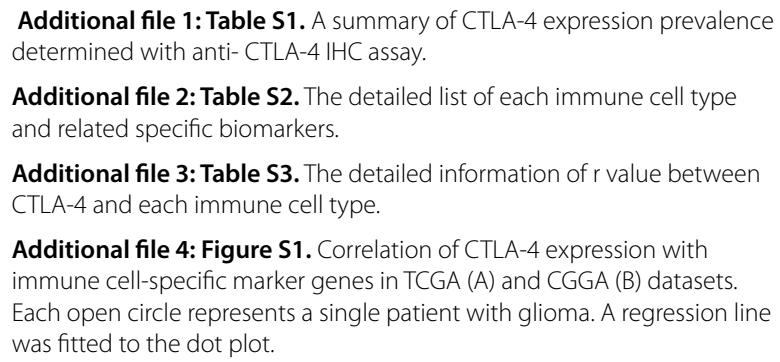

Additional file 3: Table S3. The detailed information of $r$ value between CTLA-4 and each immune cell type.

Additional file 4: Figure S1. Correlation of CTLA-4 expression with immune cell-specific marker genes in TCGA (A) and CGGA (B) datasets. Each open circle represents a single patient with glioma. A regression line was fitted to the dot plot.

\section{Abbreviations}

TCGA: The Cancer Genome Atlas; CGGA: Chinese Glioma Genome Atlas; IDH: isocitrate dehydrogenase; GBM: glioblastoma; GO: Gene Ontology; ROC: receiver operating characteristic curve; AUC: area under the curve.

\section{Acknowledgements}

We would like to thank several anonymous reviewers for their valuable comments and suggestions to improve the quality of the paper. Moreover, we want to extend our heartfelt acknowledgement to Professor Chuanbao Zhang and his group who have offered us great help and guidance in the analysis process.

\section{Authors' contributions}

$\mathrm{JH}$ and FL conceptualized and designed the study; FL led the review process, drafted the initial manuscript, and $\mathrm{JH}$ reviewed all articles and extracted data; and $\mathrm{JH}, \mathrm{FL}$, and ZL analyzed and interpreted the data. $\mathrm{XL}, \mathrm{QC}$, and $\mathrm{CL}$ helped with the revised manuscript and checked the data. All authors made substantial contributions to revising the manuscript and approved the final manuscript as submitted. ZL is responsible for the overall content. All authors read and approved the final manuscript.

\section{Funding}

This work was supported by the National Natural Science Foundation of China under grant No. 81873635; the Natural Science Foundation for Young Scientist of Hunan Province, China under Grant Nos. 2019JJ50952 and $2019 J J 50942$.

\section{Availability of data and materials}

The RNA sequencing data and related clinical information were obtained from TCGA (http://cancergenome.nih.gov/) databases and CGGA (http://www. cgga.org.cn/) databases. All other data and materials are available from the corresponding author upon reasonable request.
Ethics approval and consent to participate

The study was approved by the ethics committee of the Xiangya Hospital, Central South University (No. 2017121019).

\section{Consent for publication}

Not applicable.

\section{Competing interests}

The authors declare that they have no competing interests.

\section{Author details}

${ }^{1}$ Department of Neurosurgery, Xiangya Hospital, Central South University (CSU), 87 Xiangya Rd, Changsha 410008, Hunan, China. ${ }^{2}$ Department of Psychiatry, The Second Xiangya Hospital, Central South University, Changsha 410011, Hunan, China. ${ }^{3}$ Mental Health Institute of the Second Xiangya Hospital, Central South University, Chinese National Clinical Research Center on Mental Disorders (Xiangya), Chinese National Technology Institute on Mental Disorders, Hunan Key Laboratory of Psychiatry and Mental Health, Changsha 410011, Hunan, China. ${ }^{4}$ Intensive Care Unit, Hunan Provincial Hospital of Traditional Chinese Medicine, Zhuzhou, China.

Received: 13 October 2019 Accepted: 23 December 2019

Published online: 06 January 2020

\section{References}

1. Louveau A, Smirnov I, Keyes TJ, Eccles JD, Rouhani SJ, Peske JD, Derecki NC, Castle D, Mandell JW, Lee KS. Structural and functional features of central nervous system lymphatic vessels. Nature. 2015;523(7560):337.

2. Liu J, Albrecht AM, Ni X, Yang J, Li M. Glioblastoma tumor initiating cells: therapeutic strategies targeting apoptosis and microRNA pathways. Curr Mol Med. 2013;13(3):352-7.

3. Wang Z, Bao Z, Yan W, You G, Wang Y, Li X, Zhang W. Isocitrate dehydrogenase 1 (IDH1) mutation-specific microRNA signature predicts favorable prognosis in glioblastoma patients with IDH1 wild type. J Exp Clin Cancer Res. 2013;32:59.

4. Ouyang M, White EE, Ren H, Guo Q, Zhang I, Gao H, Yanyan S, Chen $X$, Weng $Y$, Da Fonseca A, et al. Metronomic doses of temozolomide enhance the efficacy of carbon nanotube CpG immunotherapy in an invasive glioma model. PLoS ONE. 2016;11(2):e0148139.

5. Fridman WH, Zitvogel L, Sautès-Fridman C, Kroemer G. The immune contexture in cancer prognosis and treatment. Nat Rev Clin Oncol. 2017;14(12):717.

6. Parry RV, Chemnitz JM, Frauwirth KA, Lanfranco AR, Braunstein I, Kobayashi SV, Linsley PS, Thompson CB, Riley JL. CTLA-4 and PD-1 receptors inhibit T-cell activation by distinct mechanisms. Mol Cell Biol. 2005;25(21):9543-53.

7. Huang J, Liu F, Liu Z, Tang H, Wu H, Gong Q, Chen J. Immune checkpoint in glioblastoma: promising and challenging. Front Pharmacol. 2017;8:242.

8. Liu Y, Zeng G. Cancer and innate immune system interactions: translational potentials for cancer immunotherapy. J Immunother. 2012;35(4):299.

9. Tang A, Judge TA, Nickoloff BJ, Turka LA. Suppression of murine allergic contact dermatitis by CTLA4lg. Tolerance induction of Th2 responses requires additional blockade of CD40-ligand. J Immunol. 1996;157(1):117-25.

10. Hodges TR, Ott M, Xiu J, Gatalica Z, Swensen J, Zhou S, Huse JT, de Groot J, Li S, OverwijkWW. Mutational burden, immune checkpoint expression, and mismatch repair in glioma: implications for immune checkpoint immunotherapy. Neuro-oncology. 2017;19(8):1047-57.

11. Bacolod MD, Talukdar S, Emdad L, Das SK, Sarkar D, Wang XY, Barany F, Fisher PB. Immune infiltration, glioma stratification, and therapeutic implications. Transl Cancer Res. 2016;5(Suppl 4):S652-6.

12. Doucette T, Rao G, Rao A, Shen L, Aldape K, Wei J, Dziurzynski K, Gilbert $M$, Heimberger AB. Immune heterogeneity of glioblastoma subtypes: extrapolation from the cancer genome atlas. Cancer Immunol Res. 2013;1(2):112-22.

13. Team RC. R: a language and environment for statistical computing. 2013.

14. Kolde R. Pheatmap: pretty heatmaps. R package version 2012;61. 
15. Gu Z, Gu L, Eils R, Schlesner M, Brors B. circlize implements and enhances circular visualization in R. Bioinformatics. 2014;30(19):2811-2.

16. Robin X, Turck N, Hainard A, Tiberti N, Lisacek F, Sanchez J-C, Müller M. PROC: an open-source package for $\mathrm{R}$ and $\mathrm{S}+$ to analyze and compare ROC curves. BMC Bioinformatics. 2011;12(1):77.

17. Therneau T. A package for survival analysis in S. version 2.38. 2015. Reference Source. 2017.

18. Yan H, Parsons DW, Jin G, McLendon R, Rasheed BA, Yuan W, Kos I, BatinicHaberle I, Jones S, Riggins GJ. IDH1 and IDH2 mutations in gliomas. N Engl J Med. 2009;360(8):765-73.

19. Verhaak RG, Hoadley KA, Purdom E, Wang V, Qi Y, Wilkerson MD, Miller CR, Ding L, Golub T, Mesirov JP. Integrated genomic analysis identifies clinically relevant subtypes of glioblastoma characterized by abnormalities in PDGFRA, IDH1, EGFR, and NF1. Cancer Cell. 2010;17(1):98-110.

20. Domingues P, González-Tablas M, Otero Á, Pascual D, Miranda D, Ruiz L, Sousa P, Ciudad J, Gonçalves JM, Lopes MC. Tumor infiltrating immune cells in gliomas and meningiomas. Brain Behav Immun. 2016;53:1-15.

21. Becht E, Giraldo NA, Lacroix L, Buttard B, Elarouci N, Petitprez F, Selves J, Laurent-Puig P, Sautès-Fridman C, Fridman WH. Estimating the population abundance of tissue-infiltrating immune and stromal cell populations using gene expression. Genome Biol. 2016;17(1):218.

22. Postow MA, Sidlow R, Hellmann MD. Immune-related adverse events associated with immune checkpoint blockade. N Engl J Med. 2018;378(2):158-68.

23. Larkin J, Chiarion-Sileni V, Gonzalez R, Grob JJ, Cowey CL, Lao CD, Schadendorf D, Dummer R, Smylie M, Rutkowski P. Combined nivolumab and ipilimumab or monotherapy in untreated melanoma. N Engl J Med. 2015;373(1):23-34.

24. Genoud V, Marinari E, Nikolaev SI, Castle JC, Bukur V, Dietrich P-Y, Okada $\mathrm{H}$, Walker PR. Responsiveness to anti-PD-1 and anti-CTLA-4 immune checkpoint blockade in SB28 and GL261 mouse glioma models. Oncolmmunology. 2018;7(12):e1501137.

25. Sorensen MR, Holst PJ, Steffensen MA, Christensen JP, Thomsen AR. Adenoviral vaccination combined with CD40 stimulation and CTLA-4 blockage can lead to complete tumor regression in a murine melanoma model. Vaccine. 2010;28(41):6757-64.

26. Spranger S, Koblish HK, Horton B, Scherle PA, Newton R, Gajewski TF. Mechanism of tumor rejection with doublets of CTLA-4, PD-1/PD-L1, or IDO blockade involves restored IL-2 production and proliferation of CD8+ T cells directly within the tumor microenvironment. J Immunother Cancer. 2014;2(1):3.

27. Fu T, He Q Sharma P. The ICOS/ICOSL pathway is required for optimal antitumor responses mediated by anti-CTLA-4 therapy. Cancer Res. 2011;71:5445-54.

28. Liu S, Wang Z, Wang Y, Fan X, Zhang C, Ma W, Qiu X, Jiang T. PD-1 related transcriptome profile and clinical outcome in diffuse gliomas. Oncolmmunology. 2018;7(2):e1382792.

29. Wang Z, Zhang C, Liu X, Wang Z, Sun L, Li G, Liang J, Hu H, Liu Y, Zhang W. Molecular and clinical characterization of PD-L1 expression at transcriptional level via 976 samples of brain glioma. Oncoimmunology 2016;5(11):e1196310.

30. Liu F, Huang J, Xiong Y, Li S, Liu Z. Large-scale analysis reveals the specific clinical and immune features of CD155 in glioma. Aging. 2019;11(15):5463.
31. Kaffes I, Szulzewsky F, Chen Z, Herting CJ, Gabanic B, Velázquez Vega JE, Shelton J, Switchenko JM, Ross JL, McSwain LF. Human mesenchymal glioblastomas are characterized by an increased immune cell presence compared to proneural and classical tumors. Oncolmmunology. 2019:8(11):e1655360.

32. Salvi S, Fontana V, Boccardo S, Merlo DF, Margallo E, Laurent S, Morabito A, Rijavec E, Dal Bello MG, Mora M. Evaluation of CTLA-4 expression and relevance as a novel prognostic factor in patients with non-small cell lung cancer. Cancer Immunol Immunother. 2012;61(9):1463-72.

33. Yang H, Bueso-Ramos C, DiNardo C, Estecio MR, Davanlou M, Geng Q-R, Fang Z, Nguyen M, Pierce S, Wei Y. Expression of PD-L1, PD-L2, PD-1 and CTLA4 in myelodysplastic syndromes is enhanced by treatment with hypomethylating agents. Leukemia. 2014;28(6):1280.

34. Laurent S, Queirolo P, Boero S, Salvi S, Piccioli P, Boccardo S, Minghelli S, Morabito A, Fontana V, Pietra G. The engagement of CTLA-4 on primary melanoma cell lines induces antibody-dependent cellular cytotoxicity and TNF-a production. J Transl Med. 2013;11(1):108.

35. Contardi E, Palmisano GL, Tazzari PL, Martelli AM, Fala F, Fabbi M, Kato T, Lucarelli E, Donati D, Polito L. CTLA-4 is constitutively expressed on tumor cells and can trigger apoptosis upon ligand interaction. Int J Cancer. 2005; 117(4):538-50.

36. Fong $B$, Jin R, Wang $X$, Safaee M, Lisiero DN, Yang I, Li G, Liau LM, Prins RM. Monitoring of regulatory $T$ cell frequencies and expression of CTLA-4 on T cells, before and after DC vaccination, can predict survival in GBM patients. PLoS ONE. 2012;7(4):e32614.

37. Garg AD, Vandenberk L, Van Woensel M, Belmans J, Schaaf M, Boon $\mathrm{L}$, De Vleeschouwer S, Agostinis P. Preclinical efficacy of immunecheckpoint monotherapy does not recapitulate corresponding biomarkers-based clinical predictions in glioblastoma. Oncoimmunology. 2017;6(4):e1295903

38. Saha D, Martuza RL, Rabkin SD. Macrophage polarization contributes to glioblastoma eradication by combination immunovirotherapy and immune checkpoint blockade. Cancer Cell. 2017;32(2):253-67.

39. Field CS, Hunn MK, Ferguson PM, Ruedl C, Ancelet LR, Hermans IF. Blocking CTLA-4 while priming with a whole cell vaccine reshapes the oligoclonal T cell infiltrate and eradicates tumors in an orthotopic glioma model. Oncoimmunology. 2017;7(1):e1376154.

40. Duraiswamy J, Kaluza KM, Freeman GJ, Coukos G. Dual blockade of PD-1 and CTLA-4 combined with tumor vaccine effectively restores $T$ cell rejection function in tumors. Cancer Res. 2013;73:3591-603.

41. Fan X, Quezada SA, Sepulveda MA, Sharma P, Allison JP. Engagement of the ICOS pathway markedly enhances efficacy of CTLA-4 blockade in cancer immunotherapy. J Exp Med. 2014;211(4):715-25.

42. Scala S. Molecular pathways: targeting the CXCR4-CXCL12 axisuntapped potential in the tumor microenvironment. Clin Cancer Res. 2015;21:4278-85

43. Dougall WC, Kurtulus S, Smyth MJ, Anderson AC. TIGIT and CD 96: new checkpoint receptor targets for cancer immunotherapy. Immunol Rev. 2017:276(1):112-20.

\section{Publisher's Note}

Springer Nature remains neutral with regard to jurisdictional claims in published maps and institutional affiliations.
Ready to submit your research? Choose BMC and benefit from:

- fast, convenient online submission

- thorough peer review by experienced researchers in your field

- rapid publication on acceptance

- support for research data, including large and complex data types

- gold Open Access which fosters wider collaboration and increased citations

- maximum visibility for your research: over 100M website views per year

At BMC, research is always in progress.

Learn more biomedcentral.com/submissions 